Research Paper

\title{
Prognostic role of pretreatment albumin-to-alkaline phosphatase ratio in locally advanced laryngeal and hypopharyngeal cancer: Retrospective cohort study
}

\author{
Jialing $\mathrm{Wu}^{\#}$, Kaiyun You\#, Yanhui Jiang\#, Ting Shen, Juanjuan Song, Changlong Chen ${ }^{\bowtie}$ and Yimin Liu \\ Department of Radiation Oncology, Sun Yat-Sen Memorial Hospital, Sun Yat-Sen University, Guangzhou, China. \\ \#These authors have contributed equally to this work and share first authorship. \\ $凶$ Corresponding authors: Yimin Liu \& Changlong Chen, E-mail: liuyim@mail.sysu.edu.cn; chenchlong@mail.sysu.edu.cn. Liu will handle correspondence at all stages of \\ refereeing and publication, also post-publication. Present address: 107 Yanjiang West Road, Yuexiu District, Guangzhou City, Guangdong Province, China.
}

(1) The author(s). This is an open access article distributed under the terms of the Creative Commons Attribution License (https://creativecommons.org/licenses/by/4.0/). See http://ivyspring.com/terms for full terms and conditions.

Received: 2021.04.10; Accepted: 2021.08.12; Published: 2021.08.27

\begin{abstract}
Background: This study was designed to assess the prognostic significance of pretreatment albumin-toalkaline phosphatase ratio (AAPR) in locally advanced laryngeal and hypopharyngeal cancer (LA-LHC).

Materials and Methods: The clinical data of 341 patients with locally advanced laryngeal and hypopharyngeal cancer diagnosed between March 2007 and December 2018 were retrospectively collected and analyzed. The optimal cut-off value of AAPR for evaluating DFS was determined using the ROC curve, and 0.4912 was selected. Based on pretreatment AAPR values, patients were divided into two groups (low vs. high AAPR). Survival analysis was used to investigate the survival distribution between the groups. Univariate and multivariate analyses were performed to evaluate the prognostic value of AAPR. Based on the results of the multivariate analysis, we further developed models of DFS and OS. We assigned low AAPR, N1-3, age $\geq 65$ years, and positive vascular invasion one score, respectively.

Results: Survival analysis demonstrated that the survival of patients with low and high AAPR was significantly different (low vs. high AAPR: 5-year DFS, 46.0 vs. $71.9 \%, p<0.001$; 5-year OS, 69.0 vs. 72.6\%, $p<0.001$ ). Univariate and multivariate analyses further showed that pretreatment AAPR served as an independent indicator in LA-LHC. Moreover, survival analysis showed that patients with high model score had poorer DFS and OS (5-year DFS: 58.1, 42.7, 26.9 and $9.1 \%$ of score zero, one, two, and three respectively, $\mathrm{p}<0.001$; 5-year OS: $63.0,50.3,34.1$ and $28.6 \%$ of score zero, one, two, and three respectively, $\mathrm{p}<0.001$ ).

Conclusion: Pretreatment AAPR could be an independent prognostic indicator in patients with LA-LHC. Incorporating AAPR into the risk stratification model might better categorize patients with worse oncological outcomes and support treatment strategy making.
\end{abstract}

Key words: Albumin-to-alkaline phosphatase ratio, Larynx, Hypopharynx, Prognosis, Surgery, Head and neck squamous cell carcinoma

\section{Introduction}

Of the 8.78 million cases of head and neck cancer (HNC) worldwide in 2020, with 4.44 million deaths, approximately $30 \%$ are attributed to laryngeal and hypopharyngeal cancer [1]. Among HNC, laryngeal and hypopharyngeal cancer (LHC), which pertains to the upper aerodigestive tract, is confronting the same carcinogenic substances, such as alcohol, nicotine, nitroso compounds, and HPV [2,3]. Patients with LHC possess akin treatment options and similar clinical management strategies [4,5]. More than 50\% of LHC patients are diagnosed with the locally advanced disease during their initial clinical consultation [6]. The 5-year overall survival (OS) and disease-free survival (DFS) of locally advanced laryngeal and hypopharyngeal cancer (LA-LHC) were in the range of $47.7-60.2 \%$ and $42.2-69.4 \%$, respectively, according to various publications [7-12].

Treatment options for patients with LHC include radical surgery and organ-preserving treatment modalities. Over the last few decades, radiotherapy 
and chemotherapy have gradually achieved a dominant position [12-15]. The importance of radical surgery remains, especially for patients with locally advanced stage situations, as surgery has a preferable survival rate [16]. The main surgical strategies prescribed to patients with LHC are total (or partial) laryngectomy and laryngopharyngectomy combined with cervical lymph node dissection [5]. Postoperative adjuvant therapy is administered to patients with adverse clinicopathologic features [17]. Considering the unsatisfactory oncologic outcomes of LA-LHC, the development of novel prognostic factors that can predict clinical consequences is warranted.

Patients with LA-LHC usually suffered either from tumors of the T3-4 stage or from lymph node metastasis of the N2-3 stage. Primary tumors categorized as T3-4 are larger than $4 \mathrm{~cm}$ in the greatest dimension or extensive invasion of surrounding tissues. Lymph node statuses categorized as N2-3 are larger than $3 \mathrm{~cm}$, multiple in number, or extranodal extension [18]. Both conditions resulted in an increased possibility of infection and progressive dysphagia, ultimately leading to cachexia and poor clinical outcomes. In this study, we aimed to evaluate and identify prognostic factors of the oncological outcomes of LA-LHC.

Serum albumin (ALB) is an indicator of nutritional status, liver function, renal function, and immunological function. Serum alkaline phosphatase (ALP) projects systemic inflammation and liver function. Both ALB and ALP levels were significantly associated with the prognosis of cancer patients. Thus, we hypothesized that combining ALB with ALP into one factor could better indicate the survival outcomes of patients with LA-LHC. The prognostic value of the albumin-to-alkaline phosphatase ratio (AAPR) was first reported in patients with hepatocellular carcinoma in 2015 [19]. This novel prognostic factor has been recently exploited in several malignancies (e.g., nasopharyngeal carcinoma, liver cancer, renal cell carcinoma, pancreatic ductal adenocarcinoma, etc.,) and low AAPR was associated with worse survival outcomes [20-24]. Although research on using AAPR for various malignances has been conducted, studies on the prognostic value of AAPR for LA-LHC are still scarce. Here, we performed a retrospective study on the association between AAPR and LA-LHC prognosis.

\section{Materials and methods}

\section{Patients}

Clinical data of 341 laryngeal or hypopharyngeal cancer patients diagnosed between March 2007 and December 2018 at our institution were retrospectively reviewed and collected. We included patients: (1) with eligible pretreatment serum ALB and ALP, (2) with histologically confirmed laryngeal or hypopharyngeal cancer, (3) with stage III or IV disease, and (4) older than 18 years. We excluded patients with: (1) lack of available pretreatment ALB or ALP, (2) distant metastasis, (3) stage I or II disease. A total of 341 patients were included in this study. Clinicopathological records, including age, sex, margin status, vascular invasion, surgery, adjuvant radiotherapy, adjuvant chemotherapy, TNM stage, and laboratory test results, including ALB and ALP, were collected and evaluated.

\section{Albumin-to-alkaline phosphatase ratio (AAPR)}

Patients' peripheral blood samples were extracted for laboratory examination within 2-4 weeks before the initiation of any therapy. Normal serum ALB levels were determined to 35.0-50.0 g/L. Normal serum ALP levels were determined to $45-125 \mathrm{U} / \mathrm{L}$ for male and $50-135 \mathrm{U} / \mathrm{L}$ for female. Pretreatment AAPR was calculated by dividing ALB by ALP. The optimal cut-off value of AAPR evaluating DFS was worked out by employing ROC curve, and 0.4912 was selected as it had the maximum Youden index value (sensitivity: 56.7\%; specificity: 68.7\%; Youden index: 0.254). Based on pretreatment AAPR levels, patients were classified into high AAPR group (AAPR $\geq$ 0.4912) and low AAPR group (AAPR < 0.4912 ).

\section{Clinical staging}

Before anti-tumor treatments, all patients underwent clinical assessments, including physical inspections, laboratory tests, chest radiography, electronic laryngoscopy and esophageal barium meal examination. Contrast-enhanced magnetic resonance imaging (MRI) and computed tomography (CT) of the larynx and hypopharynx used to examine the tumor size, surrounding invasion, and lymph node metastasis status were performed in selected patients. Abdominal ultrasonography and whole-body bone scan using single-photon emission computed tomography (SPECT), which aimed to detect distant metastasis, were performed in some patients.

Tumor-node-metastasis (TNM) classification was based on pretreatment examinations and postoperative pathological findings determined by the 7th edition of the American Joint Committee on Cancer (AJCC) for laryngeal and hypopharyngeal cancer.

\section{Treatment and follow up}

The treatment plan was discussed and determined by experienced otolaryngologist, oncologist, and radiologist. All patients received surgery-based combined therapy. Surgery modalities 
included larynx and hypopharynx tumor excision without cervical lymph node dissection and total (or partial) laryngectomy or laryngopharyngectomy, with or without cervical dissection.

Postoperative therapy was accomplished in patients with adverse pathologic features such as extranodal extension, positive surgery margin, pT3 or pT4, multiple positive lymph nodes, vascular invasion, etc. 153(44.3\%) patients were allocated adjuvant chemotherapy. In this group of patients, the chemotherapy scheme of TP (taxol and platinum) $(84.9 \%)$ was predominantly used, in addition to other platinum-based chemotherapy. Adjuvant radiotherapy (3D conformal RT or IMRT) was prescribed to some patients. The total doses of the tumor bed and lymphatic drainage area were 60-66Gy and 50-56Gy, respectively (1.8-2.0Gy/fraction; 5 times per week; in 6-7 weeks).

Patients were monitored every 3 months for the first 2 years, every 6 months for the subsequent 3-year, and annually thereafter.

\section{Statistical analysis}

Data statistical analyses were conducted by using SPSS 25.0 software, and two-sided P-value $\leq$ 0.05 was considered statistically significant. Chi-square test was performed to evaluate the relevance between categorical variables and AAPR. Survival curves were worked out by Kaplan-Meier method, and the significant differences of survival between groups were calculated by log-rank test. Univariate and multivariate analyses were performed to further chase down the risk factors of DFS and OS.

\section{Results}

\section{Patients' baseline characteristics}

Baseline clinical data of 341 patients with locally advanced laryngeal and hypopharyngeal cancer (LA-LHC) were retrospectively collected and analyzed. All patients enrolled in this study were East Asian. The median follow-up time was 4.67 years (range: 0.42-13.25 year). All patients were classified into two groups: low AAPR group $(\leq 0.4912)$ and high AAPR group (>0.4912). The study included 331 males and 10 females. The median age was 59 years (range, $29-95$ years). A total of $284(83.3 \%)$ patients were heavy cigarette smokers, and $160(46.9 \%)$ patients had a history of heavy drinking. In terms of the 7th edition TNM staging system of AJCC, all patients were presenting at stage III-IV. Fifty-five $(16.1 \%)$ patients were defined as T1-2 stage, and $286(83.9 \%)$ patients were classified as T3-4 stage. Concerning lymph node metastasis, the numbers of patients with N0 and N1-3 were $124(36.4 \%)$ and $217(63.6 \%)$ respectively. The mean values of ALB and ALP were 40.1 (g/L, interquartile range: $37.8-43.0 \mathrm{~g} / \mathrm{L}$ ) and $78.84(\mathrm{U} / \mathrm{L}$, interquartile range: $66-95 \mathrm{U} / \mathrm{L})$. At the last follow-up, 128 patients died, and 213 patients were alive. Recurrence was observed in 126 patients. The patients' clinical and pathological information is summarized in Table 1.

Table 1. Associations between AAPR and clinicopathological characteristics

\begin{tabular}{|c|c|c|c|}
\hline Variable & $\begin{array}{l}\text { Low level of AAPR } \\
(\mathrm{N}=139)\end{array}$ & $\begin{array}{l}\text { High level of } \\
\text { AAPR }(N=202)\end{array}$ & $P$ value \\
\hline Age, year & & & 0.482 \\
\hline$<65$ & $97(69.8 \%)$ & $148(73.3 \%)$ & \\
\hline$\geq 65$ & $42(30.2 \%)$ & $54(26.7 \%)$ & \\
\hline Sex & & & 0.482 \\
\hline male & $136(97.8 \%)$ & $195(96.5 \%)$ & \\
\hline female & $3(2.2 \%)$ & $7(3.5 \%)$ & \\
\hline T stage ${ }^{\dagger}$ & & & 0.469 \\
\hline T1-2 & $20(14.4 \%)$ & $35(17.3 \%)$ & \\
\hline T3-4 & $119(85.6 \%)$ & $167(82.7 \%)$ & \\
\hline $\mathbf{N}$ status $\dagger$ & & & 0.204 \\
\hline N0 & $45(32.4 \%)$ & $79(39.1 \%)$ & \\
\hline N1-3 & $94(67.6 \%)$ & $123(60.9 \%)$ & \\
\hline TNM stage $^{\dagger}$ & & & 0.239 \\
\hline III & $49(35.2 \%)$ & $84(41.6 \%)$ & \\
\hline IV & $90(64.8 \%)$ & $118(58.4 \%)$ & \\
\hline Site & & & 0.277 \\
\hline laryngeal & $66(47.5 \%)$ & $108(53.5 \%)$ & \\
\hline hypopharyngeal & $73(52.5 \%)$ & $94(46.5 \%)$ & \\
\hline Surgical margin & & & 0.681 \\
\hline positive & $10(7.2 \%)$ & $17(8.4 \%)$ & \\
\hline negative & $129(92.8 \%)$ & $185(91.6 \%)$ & \\
\hline Vascular invasion & & & 0.461 \\
\hline yes & $13(9.4 \%)$ & $24(11.9 \%)$ & \\
\hline no & $126(90.6 \%)$ & $178(88.1 \%)$ & \\
\hline Adjuvant radiotherapy & & & 0.781 \\
\hline yes & $101(72.7 \%)$ & $144(71.3 \%)$ & \\
\hline no & $38(27.3 \%)$ & $58(28.7 \%)$ & \\
\hline Adjuvant chemotherapy & & & 0.094 \\
\hline yes & $54(38.8 \%)$ & $97(48.1 \%)$ & \\
\hline no & $85(61.2 \%)$ & $105(51.9 \%)$ & \\
\hline Smoking history & & & 0.602 \\
\hline yes & $114(82.0 \%)$ & $170(84.2 \%)$ & \\
\hline no & $25(18.0 \%)$ & $32(15.8 \%)$ & \\
\hline Drinking history & & & 0.477 \\
\hline yes & $62(44.6 \%)$ & $98(48.5 \%)$ & \\
\hline no & $77(55.4 \%)$ & $104(51.5 \%)$ & \\
\hline
\end{tabular}

\section{Survival analysis}

Kaplan-Meier survival analysis and log-rank test demonstrated that the survival of patients with low and high AAPR was significantly different (low vs. high AAPR: DFS, 3-year, 58.6 vs. $81.2 \%$, 5-year, 46.0 vs. $71.9 \%$, p<0.001; OS, 3-year, 70.8 vs. $84.6 \%$, 5-year, 69.0 vs. $72.6 \%, \mathrm{p}<0.001$ ). Moreover, survival analysis showed that patients with low AAPR levels were significantly correlated with inferior LRFS (low vs. high AAPR, 3-year, 63.4 vs. 85.2\%, 5-year, 55.7 vs. 
$78.6 \%, \mathrm{p}<0.001)$. However, the relationship between AAPR and DMFS was not significant $(\mathrm{p}=0.140)$ (Table 2). The Kaplan-Meier curves of DFS and OS of patients with AAPR $\leq 0.4912$ and AAPR $>0.4912$ are shown in Fig. 1.


Figure 1. Kaplan-Meier curve for disease-free survival and overall survival, stratified by pretreatment AAPR, cutoff value: 0.4912 .

Table 2. Survival analysis

\begin{tabular}{|c|c|c|c|c|c|}
\hline \multirow[t]{2}{*}{ Group } & \multicolumn{2}{|c|}{ Low level of AAPR $(\mathrm{N}=139)$} & \multicolumn{2}{|c|}{ High level of AAPR $(\mathrm{N}=202)$} & \multirow[t]{2}{*}{ P value } \\
\hline & 3-year & 5-year & 3-year & 5-year & \\
\hline DFS & $58.6 \%$ & $46.0 \%$ & $81.2 \%$ & $71.9 \%$ & 0.000 \\
\hline OS & $70.8 \%$ & $69.0 \%$ & $84.6 \%$ & $72.6 \%$ & 0.000 \\
\hline LRFS & $63.4 \%$ & $55.7 \%$ & $85.2 \%$ & $78.6 \%$ & 0.000 \\
\hline DMFS & $88.1 \%$ & $77.8 \%$ & $90.6 \%$ & $83.7 \%$ & 0.140 \\
\hline
\end{tabular}

Abbreviations: OS, overall survival; DFS, disease-free survival; LRFS, local recurrence-free survival; DMFS, distant metastasis-free survival.

OS, DFS, LRFS and DMFS were calculated by Kaplan-Meier method; P value was calculated by Log-Rank test.

\section{Univariate analysis}

Univariate analysis revealed that low pretreatment AAPR was an unfavorable determinant of DFS (low vs. high AAPR, p<0.001, HR=2.370, 95\% CI 1.667-3.378) and OS (low vs. high AAPR, $\mathrm{p}<0.001$, $\mathrm{HR}=2.049,95 \%$ CI 1.443-2.901). Additionally, N status was significantly associated with both DFS and OS. Vascular invasion was associated with DFS, whereas age was correlated with OS. However, sex, T stage, margin status, adjuvant therapy, smoking history, and drinking history were not associated with survival consequences (Table 3).

\section{Multivariate analysis}

To identify the independent risk factors for DFS and OS, we further performed multivariate analysis. Multivariate analysis further confirmed that AAPR (low vs. high AAPR, DFS, $\mathrm{p}<0.001, \mathrm{HR}=2.457,95 \% \mathrm{CI}$ 1.718-3.509; OS, $\mathrm{p}<0.001, \quad \mathrm{HR}=2.016, \quad 95 \% \quad \mathrm{CI}$ 1.418-2.865), N status (N0 vs. N1-3, DFS, $\mathrm{p}=0.024$, $\mathrm{HR}=0.637,95 \%$ CI $0.431-0.942 ; \mathrm{OS}, \mathrm{p}=0.018, \mathrm{HR}=0.632$, 95\% CI 0.432-0.925) were independent prognostic factors for both DFS and OS. Besides, vascular invasion (yes vs. no, $\mathrm{p}=0.002, \mathrm{HR}=2.193,95 \%$ CI $1.348-3.571)$ and age ( $<65$ vs. $\geq 65, p=0.009, H R=0.607$, $95 \%$ CI $0.418-0.881$ ) were independent risk factors for DFS and OS, respectively (Table 4).

Table 3. Univariate analysis of DFS and OS

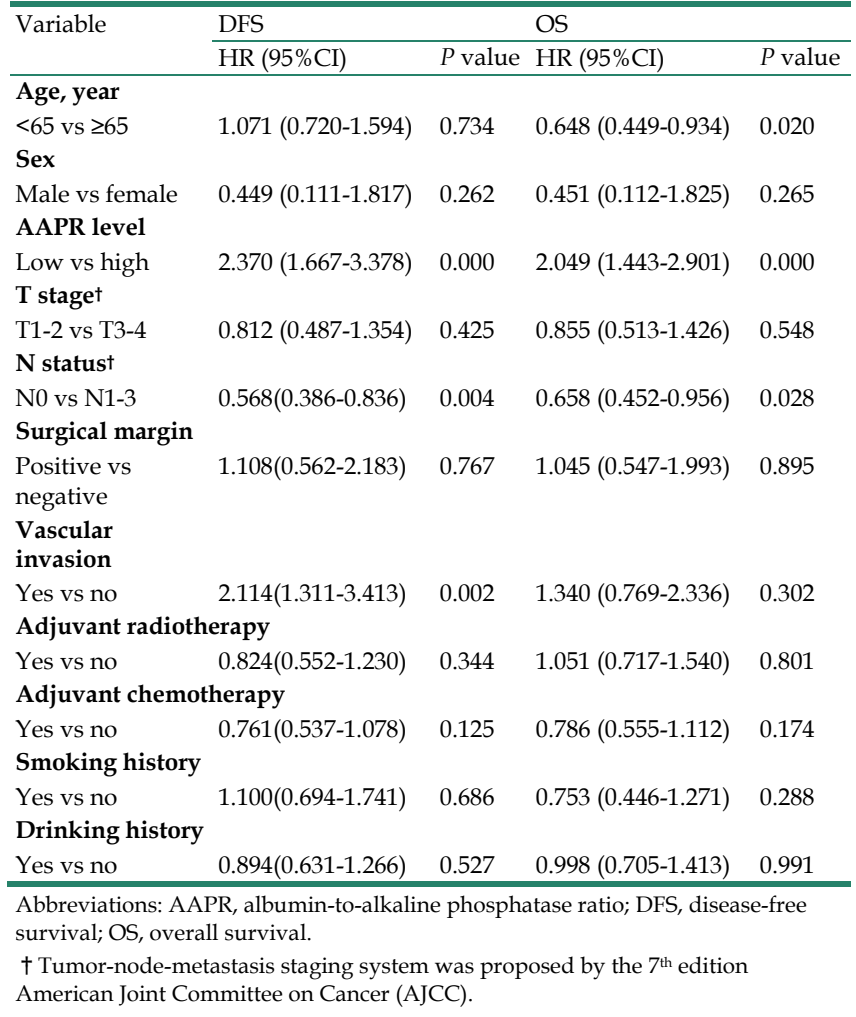

Table 4. Multivariate analysis of DFS and OS

\begin{tabular}{lllll}
\hline Variable & DFS & \multicolumn{2}{l}{ OS } & \\
\cline { 2 - 5 } & HR $(95 \% \mathrm{CI})$ & $P$ value & HR $(95 \% \mathrm{CI})$ & $P$ value \\
\hline $\begin{array}{l}\text { AAPR } \\
\text { low vs high } \\
\text { N status }\end{array}$ & $2.457(1.718-3.509)$ & 0.000 & $2.016(1.418-2.865)$ & 0.000 \\
$\begin{array}{l}\text { N0 vs N1-3 } \\
\begin{array}{l}\text { Age, year } \\
<65 \text { vs } \geq 65\end{array}\end{array}$ & $0.637(0.431-0.942)$ & 0.024 & $0.632(0.432-0.925)$ & 0.018 \\
$\begin{array}{l}\text { Vascular invasion } \\
\text { Yes vs no }\end{array}$ & NA & & $0.607(0.418-0.881)$ & 0.009 \\
\hline
\end{tabular}

Abbreviations: DFS, disease-free survival; OS, overall survival; $\mathrm{CI}$, confidence interval; HR, hazard ratio. 

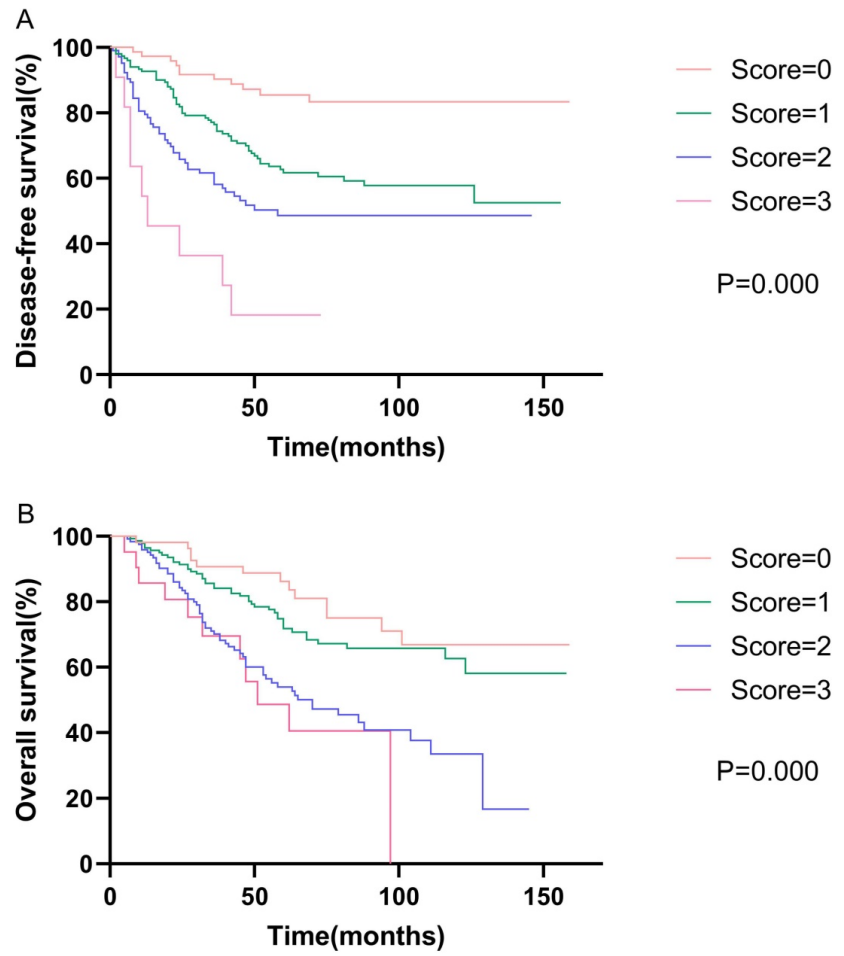

Figure 2. Kaplan-Meier curve for disease-free survival and overall survival, stratified by prognostic models of DFS and OS.

\section{Prognostic models}

Based on the results of the multivariate analysis, we further developed models of DFS and OS. The DFS model consisted of AAPR, $\mathrm{N}$ status, and vascular invasion, while the OS model consisted of AAPR, N status, and age. We assigned low AAPR, N1-3, old age ( $\geq 65$ years), and positive vascular invasion, one score respectively. Survival analysis showed that patients with high model scores had poorer DFS and OS. The 5-year disease-free survival rates were $58.1 \%, 42.7 \%$, $26.9 \%$, and $9.1 \%$ for scores of zero, one, two, and three, respectively $(p<0.001)$. The 5-year overall survival rates were $63.0 \%, 50.3 \%, 34.1 \%$, and $28.6 \%$ for scores of zero, one, two, and three, respectively $(\mathrm{p}<0.001)$. The Kaplan-Meier curves of prognostic models of DFS and OS are presented in Fig. 2.

\section{Discussion}

Laryngeal and hypopharyngeal cancers have frequently been investigated as a single entity and shared similar treatment strategies $[4,7,8,10,11,14]$. To the best of our knowledge, this is the first study to investigate the prognostic value of pretreatment AAPR in locally advanced laryngeal and hypopharyngeal cancer (LA-LHC). In this study, we found that low AAPR was associated with less favorable survival outcomes, and pretreatment AAPR served as an independent indicator in LA-LHC.

AAPR is derived from serum ALB and ALP, which can be easily and reproducibly accessed and extracted from blood tests. The relatively low levels of ALB or hypoalbuminemia in patients with cancer can be explained by: (1) stress response provoked by illnesses, (2) low protein intake, and (3) intercurrent infections [25]. Researchers have found a correlation between low serum ALB level and immunological dysfunction [26]. The resulting diminished immune function fuel the growth of tumors and further jeopardize the efficacy of anti-cancer treatment. One study examined the clinical utility of pretreatment serum ALB in patients with head and neck squamous cell carcinoma (HNSCC). The results showed that there was a relationship between low pretreatment albumin and increased wound infection and inferior OS.

Moreover, this relationship was most prominent in patients with upper aerodigestive squamous cell carcinoma [27]. Parallel to what had been observed, Lim et al. also reported that low pretreatment serum ALB significantly increased the risk of tumor progression and cancer-related death [28]. Another study investigated the prognostic role of serum ALB in patients with advanced HNSCC who underwent surgery and flap reconstruction and determined that preoperative hypoalbuminemia was a poor prognostic indicator in this population [29]. Also, studies have shown that low serum ALB is an unfavorable determinant in other malignancies, including oral, breast, and vulvar cancers [30-32].

Alkaline phosphatase (ALP) is a serum metalloenzyme. The elevation of ALP projects the presence of hepatobiliary disease and liver and bone metastases. ALP is an indicator of hepatobiliary tract obstruction[33] and elevated osteoblastic activity[34], and thus serving as a very subtle predictor of liver and bone metastases in patients with cancer. The prognostic role of ALP has been observed in many studies, which have consistently revealed that ALP is an unfavorable factor, as well as an indicator of cancer recurrence and distant metastasis [35-37]. Specifically, study disclosed that high pretreatment ALP was significantly correlated with poor survival in patients with laryngeal cancer [38].

Overall, pretreatment AAPR reflected primary tumor load, inflammatory condition, and nutrition condition. AAPR appeared to perform better than ALP and ALB alone in predicting the survival outcomes of cancer patients. A meta-analysis conducted by Tian et al. evaluated the possible utility of AAPR in solid cancers, and showed that low pretreatment AAPR was associated with worse OS in solid cancers, such as lung cancer, hepatocellular carcinoma, pancreatic ductal adenocarcinoma, and nasopharyngeal carcinoma [23]. Burgeoning eviden- 
ces suggested the important prognostic value of AAPR $[20,21,39,40]$, which were consistent with our findings.

Additionally, our findings revealed that $\mathrm{N}$ status was an independent prognostic factor for both DFS and OS, consistent with previous reports [41, 42]. Vascular invasion and age were independent risk factors for DFS and OS, respectively. Vascular invasion is associated with lymph node metastasis $[43,44]$ and a well-established survival predictor in various cancers, including esophageal, upper urinary tract urothelial, oral tongue squamous cell, and adenoid cystic carcinoma [43-46]. In our study, we chose 65 years as the inflection point; however, Abrahão et al. selected 71 years and reported that patients $\geq 71$ years were associated with increased mortality risk [47]. Our study showed that heavy alcohol consumption and tobacco use were prevalent among head and neck cancer patients. Studies have demonstrated that tobacco smoking is associated with mortality in head and neck cancers [48, 49]. However, our study failed to support this relationship, and we speculated that the retrospective nature and relatively small size of our study account for the negative result. Nevertheless, our finding of alcohol consumption was consistent with previous literature [48].

More intriguingly, we constructed models that incorporated AAPR, N status, and vascular invasion for DFS and contained AAPR, N status, and age for OS. Survival analysis revealed that these models improved the stratification of patients with various risks. Incorporating these models into clinical use may help clinicians in treatment making.

Some limitations of this study should be noted. First, the retrospective nature of this study inevitably dampened its utility. Second, AAPR is based on ALB and ALP. Since abnormal ALB and ALP can result from various non-cancer situations, and thus AAPR is not a cancer-specific biomarker. Both ALB and ALP abnormalities can affect AAPR. The cause of abnormal ALB and ALP include malnutrition, benign liver disease, and renal dysfunction, among others. Exploiting this intriguing prognostic factor requires further diligence and robust studies. Third, our study focused on patients who received surgery-based treatment, which was the main treatment option for patients with LA-LHC in our institution; thus, our results might fail to extend to patients who underwent definitive chemoradiotherapy. In addition, external validation of our main findings and conclusions is required. Moreover, since the patients collected and evaluated in this study were predominantly males, the results did not extrapolate well to female patients with LA-LHC.

\section{Conclusion}

For the first time, our study sheds new light on the prognostic impact of AAPR in patients with LA-LHC. Our results showed that pretreatment AAPR was an independent prognostic factor in patients with LA-LHC. Incorporating AAPR into stratification models may better forecast the oncological consequences of patients with LA-LHC. More well-designed, prospective and multi-center studies are required to further verify the clinical value of AAPR.

\section{Acknowledgements}

\section{Author's contributions}

JLW: was responsible for writing-original draft, KYY and YHJ: were responsible for writing-review, TS: was responsible for revision of the draft, JJS: was responsible for data collection and analysis, CLC and YML: were responsible for the study design. The final manuscript was approved by all authors who granted to be accountable for the content of this manuscript.

\section{Ethics Statement}

This study was conducted under the principle of the Institutional Review Board at Sun Yat-Sen Memorial Hospital, Sun Yat-Sen University (protocol code SYSEC-KY-KS-2021-036).

\section{Funding}

This study was funded by the Natural Science Foundation of Guangdong Province, China (No. 2016A030313361) and Natural Science Foundation for Young Scholar of China (No. 82003071).

\section{Competing Interests}

The authors have declared that no competing interest exists.

\section{References}

1. Sung H, Ferlay J, Siegel RL, Laversanne M, Soerjomataram I, Jemal A, et al. Global cancer statistics 2020: GLOBOCAN estimates of incidence and mortality worldwide for 36 cancers in 185 countries. CA: a cancer journal for clinicians. 2021;71(3):209-249.

2. Hashim D, Genden E, Posner M, Hashibe M, Boffetta P. Head and neck cancer prevention: from primary prevention to impact of clinicians on reducing burden. Ann Oncol. 2019; 30: 744-56.

3. Bates JE, Morris CG, Hitchcock KE, Dziegielewski PT, Mendenhall WM, Amdur RJ. Locally advanced hypopharyngeal and laryngeal cancer: Influence of HPV status. Radiother Oncol. 2019; 140: 6-9.

4. Lee NY, O'Meara W, Chan K, Della-Bianca C, Mechalakos JG, Zhung J, et al. Concurrent chemotherapy and intensity-modulated radiotherapy for locoregionally advanced laryngeal and hypopharyngeal cancers. Int J Radiat Oncol Biol Phys. 2007; 69: 459-68.

5. Smith RB. Surgery in the management of laryngeal and hypopharyngeal carcinoma. Int J Radiat Oncol Biol Phys. 2007; 69: S28-30.

6. Posner MR, Norris CM, Wirth LJ, Shin DM, Cullen KJ, Winquist EW, et al. Sequential therapy for the locally advanced larynx and hypopharynx cancer subgroup in TAX 324: survival, surgery, and organ preservation. Ann Oncol. 2009; 20: 921-7.

7. Yang CJ, Kim DY, Lee JH, Roh JL, Choi SH, Nam SY, et al. Prognostic value of total tumor volume in advanced-stage laryngeal and hypopharyngeal carcinoma. J Surg Oncol. 2013; 108: 509-15. 
8. Meulemans J, Debacker J, Demarsin H, Vanclooster C, Neyt P, Mennes T, et al Oncologic Outcomes After Salvage Laryngectomy for Squamous Cell Carcinoma of the Larynx and Hypopharynx: A Multicenter Retrospective Cohort Study. Ann Surg Oncol. 2020;28(3):1751-1761.

9. Su X, He HC, Ye ZL, Zhou DL, Liu Q, Yang XH, et al. A 10-Year Study on Larynx Preservation Compared With Surgical Resection in Patients With Locally Advanced Laryngeal and Hypopharyngeal Cancers. Front Oncol. 2020; 10: 535893

10. Al-Mamgani A, Navran A, Walraven I, Schreuder WH, Tesselaar MET, Klop WMC. Organ-preservation (chemo)radiotherapy for T4 laryngeal and hypopharyngeal cancer: is the effort worth? Eur Arch Otorhinolaryngol. 2019; 276: 575-83.

11. Janoray G, Pointreau Y, Alfonsi M, Sire C, Geoffrois L, de Raucourt D, et al. Induction chemotherapy followed by cisplatin or cetuximab concomitant to radiotherapy for laryngeal/hypopharyngeal cancer: Long-term results of the TREMPLIN randomised GORTEC trial. Eur J Cancer. 2020; 133: 86-93.

12. Chung EJ, Jeong WJ, Jung YH, Kwon SK, Kwon TK, Ahn SH, et al. Long-term oncological and functional outcomes of induction chemotherapy followed by (chemo)radiotherapy vs definitive chemoradiotherapy vs surgery-based therapy in locally advanced stage III/IV hypopharyngeal cancer: Multicenter review of 266 cases. Oral Oncol. 2019; 89: 84-94

13. Denaro N, Russi EG, Lefebvre JL, Merlano MC. A systematic review of current and emerging approaches in the field of larynx preservation. Radiother Oncol. 2014; 110: 16-24

14. Argiris A, Lefebvre JL. Laryngeal Preservation Strategies in Locally Advanced Laryngeal and Hypopharyngeal Cancers. Front Oncol. 2019; 9: 419.

15. Takes RP, Strojan P, Silver CE, Bradley PJ, Haigentz M, Jr., Wolf GT, et al. Current trends in initial management of hypopharyngeal cancer: the declining use of open surgery. Head Neck. 2012; 34: 270-81.

16. Lin Z, Lin H, Chen $Y, X u Y$, Chen X, Fan H, et al. Long-term survival trend after primary total laryngectomy for patients with locally advanced laryngeal carcinoma. J Cancer. 2021; 12: 1220-30.

17. Machiels JP, Rene Leemans C, Golusinski W, Grau C, Licitra L, Gregoire V, et al. Squamous cell carcinoma of the oral cavity, larynx, oropharynx and hypopharynx: EHNS-ESMO-ESTRO Clinical Practice Guidelines for diagnosis, treatment and follow-up. Ann Oncol. 2020; 31: 1462-75.

18. Chow LQM. Head and Neck Cancer. N Engl J Med. 2020; 382: 60-72

19. Chan AW, Chan SL, Mo FK, Wong GL, Wong VW, Cheung YS, et al. Albumin-to-alkaline phosphatase ratio: a novel prognostic index for hepatocellular carcinoma. Dis Markers. 2015; 2015: 564057.

20. Kim JS, Keam B, Heo DS, Han DH, Rhee CS, Kim JH, et al. The Prognostic Value of Albumin-to-Alkaline Phosphatase Ratio before Radical Radiotherapy in Patients with Non-metastatic Nasopharyngeal Carcinoma: A Propensity Score Matching Analysis. Cancer Res Treat. 2019; 51: 1313-23.

21. Xia A, Chen Y, Chen J, Pan Y, Bao L, Gao X. Prognostic value of the albumin-to-alkaline phosphatase ratio on urologic outcomes in patients with non-metastatic renal cell carcinoma following curative nephrectomy. J Cancer. 2019: 10: 5494-503.

22. Zhang $\mathrm{K}$, Dong $\mathrm{S}$, Jing $\mathrm{YH}$, Gao HF, Chen LY, Hua YQ, et al. Albumin-to-alkaline phosphatase ratio serves as a prognostic indicator in unresectable pancreatic ductal adenocarcinoma: a propensity score matching analysis. BMC Cancer. 2020; 20: 541.

23. Tian G, Li G, Guan L, Yang Y, Li N. Pretreatment albumin-to-alkaline phosphatase ratio as a prognostic indicator in solid cancers: A meta-analysis with trial sequential analysis. Int J Surg. 2020; 81: 66-73.

24. Li H, Wang L, Chen L, Zhao H, Cai J, Yao J, et al. Prognostic Value of Albumin-to-Alkaline Phosphatase Ratio in Hepatocellular Carcinoma Patients Treated with Liver Transplantation. J Cancer. 2020; 11: 2171-80.

25. Serum albumin and mortality. Lancet. 1990; 335: 348-51

26. Dowd PS, Heatley RV. The influence of undernutrition on immunity. Clin Sci (Lond). 1984; 66: 241-8

27. Danan D, Shonka DC, Jr, Selman Y, Chow Z, Smolkin ME, Jameson MJ. Prognostic value of albumin in patients with head and neck cancer. Laryngoscope. 2016; 126: 1567-71.

28. Lim WS, Roh JL, Kim SB, Choi SH, Nam SY, Kim SY. Pretreatment albumin level predicts survival in head and neck squamous cell carcinoma. Laryngoscope. 2017; 127: E437-E42.

29. Tsai MH, Chuang HC, Lin YT, Lu H, Chen WC, Fang FM, et al. Clinical impact of albumin in advanced head and neck cancer patients with free flap reconstruction-a retrospective study. PeerJ. 2018; 6: e4490.

30. Bekos C, Polterauer S, Seebacher V, Bartl T, Joura E, Reinthaller A, et al. Pre-operative hypoalbuminemia is associated with complication rate and overall survival in patients with vulvar cancer undergoing surgery. Arch Gynecol Obstet. 2019; 300: 1015-22.

31. Lee JI, Kwon M, Roh JL, Choi JW, Choi SH, Nam SY, et al. Postoperative hypoalbuminemia as a risk factor for surgical site infection after oral cancer surgery. Oral Dis. 2015; 21: 178-84.

32. Fujii T, Tokuda S, Nakazawa Y, Kurozumi S, Obayashi S, Yajima R, et al. Implications of Low Serum Albumin as a Prognostic Factor of Long-term Outcomes in Patients With Breast Cancer. In vivo. 2020; 34: 2033-6.

33. Keshaviah A, Dellapasqua S, Rotmensz N, Lindtner J, Crivellari D, Collins J, et al. CA15-3 and alkaline phosphatase as predictors for breast cancer recurrence: a combined analysis of seven International Breast Cancer Study Group trials. Ann Oncol. 2007; 18: 701-8.
34. Karhade AV, Thio $\mathrm{O}$ Kuverji M, Ogink PT, Ferrone ML, Schwab JH. Prognostic value of serum alkaline phosphatase in spinal metastatic disease. Br J Cancer. 2019; 120: 640-6.

35. Yu MC, Chan KM, Lee CF, Lee YS, Eldeen FZ, Chou HS, et al. Alkaline phosphatase: does it have a role in predicting hepatocellular carcinoma recurrence? J Gastrointest Surg. 2011; 15: 1440-9.

36. Namikawa T, Ishida N, Tsuda S, Fujisawa K, Munekage E, Iwabu J, et al. Prognostic significance of serum alkaline phosphatase and lactate dehydrogenase levels in patients with unresectable advanced gastric cancer. Gastric Cancer. 2019; 22: 684-91.

37. Hung HY, Chen JS, Chien Y, Tang R, Hsieh PS, Wen S, et al. Preoperative alkaline phosphatase elevation was associated with poor survival in colorectal cancer patients. Int J Colorectal Dis. 2017; 32: 1775-8.

38. Chen L, Zeng H, Yang J, Lu Y, Zhang D, Wang J, et al. Survival and prognostic analysis of preoperative inflammatory markers in patients undergoing surgical resection for laryngeal squamous cell carcinoma. BMC Cancer. 2018; 18: 816.

39. Zhang F, Lu SX, Hu KS, Gan YH, Chen Y, Ge NL, et al. Albumin-to-alkaline phosphatase ratio as a predictor of tumor recurrence and prognosis in patients with early-stage hepatocellular carcinoma undergoing radiofrequency ablation as initial therapy. Int J Hyperthermia. 2021; 38: 1-10.

40. Acikgoz Y, Bal O, Dogan M. Albumin-to-Alkaline Phosphatase Ratio: Does It Predict Survival in Grade 1 and Grade 2 Neuroendocrine Tumors? Pancreas. 2021; 50: 111-7.

41. Xing Y, Zhang J, Lin H, Gold KA, Sturgis EM, Garden AS, et al. Relation between the level of lymph node metastasis and survival in locally advanced head and neck squamous cell carcinoma. Cancer. 2016; 122: 534-45.

42. Ho AS, Kim S, Tighiouart M, Gudino C, Mita A, Scher KS, et al. Association of Quantitative Metastatic Lymph Node Burden With Survival in Hypopharyngeal and Laryngeal Cancer. JAMA Oncol. 2018; 4: 985-9.

43. Habu N, Imanishi Y, Kameyama K, Shimoda M, Tokumaru Y, Sakamoto K, et al. Expression of Oct3/4 and Nanog in the head and neck squamous carcinoma cells and its clinical implications for delayed neck metastasis in stage I/II oral tongue squamous cell carcinoma. BMC Cancer. 2015; 15: 730.

44. Yang J, Lu Z, Li L, Li Y, Tan Y, Zhang D, et al. Relationship of lymphovascular invasion with lymph node metastasis and prognosis in superficial esophageal carcinoma: systematic review and meta-analysis. BMC Cancer. 2020; 20: 176.

45. Martins-Andrade B, Dos Santos Costa SF, Sant'ana MSP, Altemani A, Vargas PA, Fregnani ER, et al. Prognostic importance of the lymphovascular invasion in head and neck adenoid cystic carcinoma: A systematic review and meta-analysis. Oral Oncol. 2019; 93: 52-8.

46. Saito K, Kawakami S, Fujii Y, Sakura M, Masuda H, Kihara K. Lymphovascular invasion is independently associated with poor prognosis in patients with localized upper urinary tract urothelial carcinoma treated surgically. J Urol. 2007; 178: 2291-6; discussion 6.

47. Abrahao R, Anantharaman D, Gaborieau V, Abedi-Ardekani B, Lagiou P, Lagiou A, et al. The influence of smoking, age and stage at diagnosis on the survival after larynx, hypopharynx and oral cavity cancers in Europe: The ARCAGE study. Int J Cancer. 2018; 143: 32-44.

48. Beynon RA, Lang S, Schimansky S, Penfold CM, Waylen A, Thomas SJ, et al. Tobacco smoking and alcohol drinking at diagnosis of head and neck cancer and all-cause mortality: Results from head and neck 5000, a prospective observational cohort of people with head and neck cancer. Int J Cancer. 2018; 143: 1114-27.

49. Du E, Mazul AL, Farquhar D, Brennan P, Anantharaman D, Abedi-Ardekani B, et al. Long-term Survival in Head and Neck Cancer: Impact of Site, Stage, Smoking, and Human Papillomavirus Status. Laryngoscope. 2019; 129: 2506-13. 